\title{
A Fuzzy Probability-based Markov Chain Model for Electric Power Demand Forecasting of Beijing, China
}

\author{
Xiaonan Zhou', Ye Tang ${ }^{1}$, Yulei Xie ${ }^{1}$, Yalou $\mathrm{Li}^{2}$, Hongliang Zhang ${ }^{3}$ \\ ${ }^{1}$ Key Laboratory of Regional Energy System Optimization, Ministry of Education, \\ North China Electric Power University, Beijing, China \\ ${ }^{2}$ China Electric Power Research Institute, Beijing, China \\ ${ }^{3}$ Electric Power Research Institute of Guangdong Grid Corporation, Guangzhou, China \\ Email: weili819@yahoo.com.cn,zhouxiaonan130@yahoo.com.cn
}

Received December, 2012

\begin{abstract}
In this study, a fuzzy probability-based Markov chain model is developed for forecasting regional long-term electric power demand. The model can deal with the uncertainties in electric power system and reflect the vague and ambiguous during the process of power load forecasting through allowing uncertainties expressed as fuzzy parameters and discrete intervals. The developed model is applied to predict the electric power demand of Beijing from 2011 to 2019. Different satisfaction degrees of fuzzy parameters are considered as different levels of detail of the statistic data. The results indicate that the model can reflect the high uncertainty of long term power demand, which could support the programming and management of power system. The fuzzy probability Markov chain model is helpful for regional electricity power system managers in not only predicting a long term power load under uncertainty but also providing a basis for making multi-scenarios power generation/development plans.
\end{abstract}

Keywords: Fuzzy Probability; Markov Chain Model; Power Load Prediction; Satisfaction Degree; Uncertainty

\section{Introduction}

Electric power is one of the most usual and important energy in human life and economic development. With the incensement of urbanization and the improvement of people's living standard, electric power demand in industries, commerce and people's daily is on the increase during a long period. Thus, the electric power industry must take various actions (e.g. power expansion and transmission expansion) to meet the user's demand. However, there are many uncertain and limited factors that exist in many system parameters and their interrelationships, including the influence of weather, nature environment, human activities and economic structural adjustment. All of those would lead to the uncertainty in long term power load forecasting and the versatility of electric power demand in future. Therefore, it is desired to develop an effective method to forecast long-term power load under considering the uncertainties of electric power system.

Previously, a variety of forecasting models of electric power load have been developed, such as grey Markov model[1], artificial neutral model[2], recurrent support vector machines with genetic algorithms (RSVMG) model[3], hybrid ellipsoidal fuzzy systems (HEFST) [4] and adaptive network based fuzzy inference system
(ANFIS) [5]. All of these models attempt to minimize the forecasting error, but have less ability to reflect different possible of power demand caused by uncertainty, especially the economic structural adjustment in developing countries (e.g. China). The different possible of power demand is more obvious after a long time (more than 5 years). Therefore, it is desired to develop a set which include different possible of power demand in power load forecasting.

In this study, the objective is to develop a fuzzy probability based Markov chain model to predict long term regional electric power demand. The model is coupled with fuzzy probability and discrete interval method to Markov chain in order to deal with the vagueness and uncertainty in system parameters and their interrelationships. The model can reflect the different possible of power demand and provide information to a long term power system programming. The model is applied to the case of Beijing. The power load of Beijing is forecasted in different satisfaction degrees.

\section{Fuzzy Probability Markov Chain Model}

\subsection{Markov Chain}

A Markov chain is a sequence of random variables with 
Markov property, in other words, the present state only dependents on the last state, and doesn't depend on the states before the last state. Let $X_{t}$ denotes a random variable which representing the state of a system at time $t$, where $t=0,1,2, \ldots$ If $X_{t+1}$ only depends on the state of $X_{t}$, and does not depend on the states before $X_{t}$, formally

$$
\begin{aligned}
& P\left(X_{n+1}=x \mid X_{1}=x_{1}, X_{2}=x_{2}, \cdots, X_{n}=x_{n}\right) \\
& =P\left(X_{n+1}=x_{n+1} \mid X_{n}=x_{n}\right)
\end{aligned}
$$

We can say $X_{t}$ with Markov property, and the series $X_{t}$ is a Markov chain. If

$$
P\left(X_{n+1}=x_{n+1} \mid X_{n}=x_{n}\right)=P\left(X_{n}=x_{n} \mid X_{n-1}=x_{n-1}\right),
$$

$X_{t}$ is a stationary Markov chain (or time-homogeneous Markov chain). Let $p_{i j}$ denotes the probability that the system is in a state $\mathrm{j}$ at time $t+1$ given the system is in state $i$ at time $t$. If the system has a finite number of states, $1,2, \cdots s$, the stationary Markov chain can be defined by a transfer probability matrix[6]:

$$
P=\left[\begin{array}{cccc}
p_{11} & p_{12} & \cdots & p_{1 s} \\
p_{21} & p_{22} & \cdots & p_{2 s} \\
\vdots & \vdots & \vdots & \vdots \\
p_{s 1} & p_{s 2} & \cdots & p_{s s}
\end{array}\right]
$$

The transfer probability matrix of a stationary Markov chain can be generated from the observations of the system state in the past. Provided with the observations of the system state $X_{0}, X_{1}, X_{2}, \cdots, X_{n}$, at time $t=0, \ldots, N-1$, we can get the transfer probability matrix as follow[7]

$$
P_{i j}=\frac{N_{i j}}{N_{i}}
$$

where $N_{i j}$ is the number of observation pairs $X_{t}$ and $X_{t+1}$ with $X_{t}$ in state $i$ and $X_{t+1}$ in state $j ; N_{i}$ is the number of observation pairs $X_{t}$ and $X_{t+1}$ with $X_{t}$ in state $i$ and $X_{t+1}$ in any state.

\subsection{Fuzzy Probability Markov Chain Model}

Let $D E_{t}$ denotes the electric power load in period $t$, and $R_{t}$ denotes the growth rate of power load in period $t$. $R_{t}$ can be divided into three states $r_{1}^{ \pm}, r_{2}^{ \pm}$and $r_{3}^{ \pm}$. The power load in the period before the first programming period is $D E_{0}$ and its growth rate is in state $r_{m}^{ \pm}$

The power load in period $t$ :

$$
D E_{t}^{ \pm}=D E_{0} \prod_{i=1}^{t}\left(1+R_{i}^{ \pm}\right)
$$

$R_{t}$ is composed of 3 states, so there are $3^{t}$ probabilities of the power load in period $t$ :

$$
\begin{aligned}
& D E_{1 h}^{ \pm}=D E_{0}\left(1+\mathrm{r}_{h}^{ \pm}\right) h=1,2,3 ; \\
& D_{t+1,3 \times \mathrm{h}-2}^{ \pm}=D_{t h}^{ \pm} \cdot r_{1}^{ \pm} t=1,2,3 \cdots ; h=1,2, \cdots 3^{t} ; \\
& D_{t+1,3 \times \mathrm{h}-1}^{ \pm}=D_{t h}^{ \pm} \cdot r_{2}^{ \pm} t=1,2,3 \cdots ; h=1,2, \cdots 3^{t} ; \\
& D_{t+1,3 \times \mathrm{h}}^{ \pm}=D_{t h}^{ \pm} \cdot r_{3}^{ \pm} \quad t=1,2,3 \cdots ; h=1,2, \cdots 3^{t} ;
\end{aligned}
$$

Transfer probability matrix:

$$
\begin{gathered}
P=\left[\begin{array}{lll}
p_{11} & p_{12} & p_{13} \\
p_{21} & p_{22} & p_{23} \\
p_{31} & p_{32} & p_{33}
\end{array}\right] \\
P_{i j}=\frac{N_{i j}}{N_{i}}
\end{gathered}
$$

where $N_{i j}$ is the number of observation pairs $R_{t}$ and $R_{t+1}$ with $R_{t}$ in state $i$ and $R_{t}$ in state $j$ in historical data; $N_{i}$ is the number of state $i$ occurs in historical data except the last period.

However, the probabilities gotten from historical data are approximate value which can not reflect the real transport probability. The actual transfer probabilities distribute in the range around the statistics (It can be assumed that the range is from 0.5 times to 1.5 times of the statistic). Assume that the transfer probabilities are triangular fuzzy numbers, the satisfaction degree is 1 when the transport probability is the statistic value, and the satisfaction degree is 0 when the transport probability is one of the boundaries of the range.

When the satisfaction is higher than $u \quad(0 \leq u \leq 1)$, the lower bound:

$$
P_{i j}^{-}=P_{i j}-(1-u) \times\left(P_{i j}-0.5 P_{i j}\right)
$$

the upper bound:

$$
P_{i j}^{+}=P_{i j}+(1-u) \times\left(1.5 P_{i j}-P_{i j}\right)
$$

The transfer probability matrix:

$$
P=\left[\begin{array}{ccc}
p_{11}^{ \pm} & p_{12}^{ \pm} & p_{13}^{ \pm} \\
p_{21}^{ \pm} & p_{22}^{ \pm} & p_{23}^{ \pm} \\
p_{31}^{ \pm} & p_{32}^{ \pm} & p_{33}^{ \pm}
\end{array}\right]
$$

T step transfer probability matrix: $Q_{t}=P^{t}$, the third row of every $\mathrm{Q}_{\mathrm{t}}$ composed to a new matrix $X_{t j}, x_{t j}$ is the probability of state $j$ occurring in period $t$.

There are $3^{t}$ probabilities of the power load in period $t$, let $\operatorname{Pr}_{t h}^{ \pm}$denotes the probability of demand scenario $h$ in period t. It is obviously that when $t=1$

$$
\operatorname{Pr}_{11}^{ \pm}=p_{m 1}^{ \pm} ; \operatorname{Pr}_{12}^{ \pm}=p_{m 2}^{ \pm} ; \operatorname{Pr}_{13}^{ \pm}=p_{m 3}^{ \pm} ;
$$

when $t \geq 2$, according to Bayes formula

$$
\operatorname{Pr}_{t+1,3 \times h-2}^{ \pm}=\operatorname{Pr}_{t h}^{ \pm} \cdot p_{k, 1}^{ \pm} \quad t=2,3 \cdots ; h=1,2, \cdots 3^{t} ;
$$




$$
\begin{aligned}
& \operatorname{Pr}_{t+1,3 \times \mathrm{h}-1}^{ \pm}=\operatorname{Pr}_{t h}^{ \pm} \cdot p_{k, 2}^{ \pm} \quad t=2,3 \cdots ; h=1,2, \cdots 3^{t} ; \\
& \operatorname{Pr}_{t+1,3 \times \mathrm{h}}^{ \pm}=\operatorname{Pr}_{t h}^{ \pm} \cdot p_{k, 3}^{ \pm} \quad t=2,3 \cdots ; h=1,2, \cdots 3^{t} ;
\end{aligned}
$$

where $k$ is the remainder of a divided by $3(k=3$ when 3 divides $h$ ).

\section{Case Study}

Beijing, located in the northern part of the northern China plain (39 $56^{\prime} \mathrm{N}, 116^{\circ} 20^{\prime} \mathrm{E}$ ), is the capital of China, with a large population and a rapid economic development. From 1980s', the electric power consumption grows rapidly. Figure 1 shows the power load of Beijing from 1978 to 2010 . The average growth rate of the last 20 years was $8.40 \%$, and of the last 10 years was $7.31 \%$. The power load reaches $809.90 \times 10^{8} \mathrm{KWh}$ in 2010, $9.57 \%$ more than 2009. It is 10.09 times larger than 1978.

As the fast development of economy and the improvement of residents, the power demand of Beijing keeps increasing rapidly, especially commercial and domestic consumption. For example the commercial power consumption is $16.21 \times 10^{9}$ KWh in $2004,31.14 \times 10^{9}$ KWh in 2009 and increased $92.07 \%$ more than 2004. On the other hand, the power demand of Beijing is influenced by some uncertain factors, such as the growth rate of industry and economy, the relationship between power demand and economy.

In this study, in order to fitting the long term power system programming, 3 years are defined as a planning period, and three periods are forecasted. The first period is from 2011 to 2013, the second period is from 2014 to 2016, and the third period is from 2017 to 2019. The historical data from 1978 to 2010 is divided into 11 periods. The power load in every period is represented by upper bound and lower bound, as shown in Figure 2. Figure 3 displays the growth rate of the power load from 1978 to 2010. As shown in Figure 3, the growth rate changes in the value of several intervals. In this study, the growth rate $R_{t}$ was divided into 3 states [0.14, 0.22], $[0.22,0.30]$, and $[0.30,0.38]$, denoted as low, medium

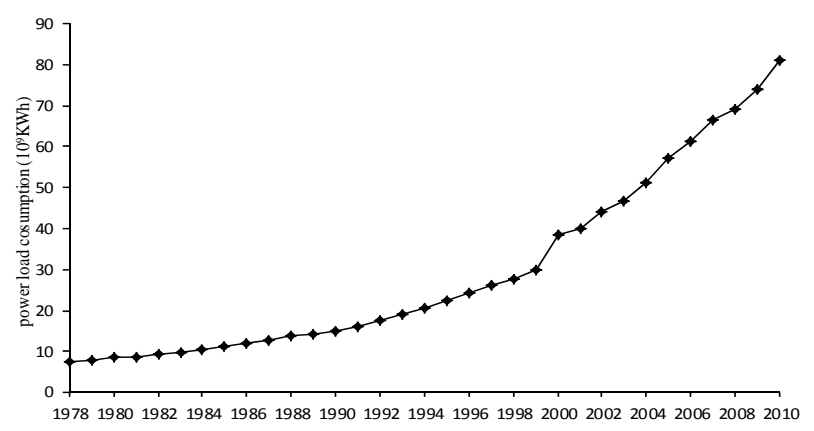

Figure 1. The power load consumption of Beijing.

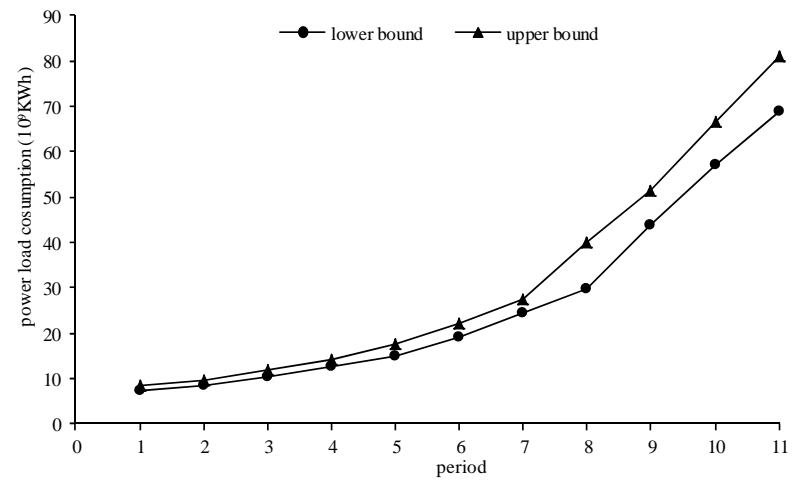

Figure 2. Power load consumption of the last 11periods.

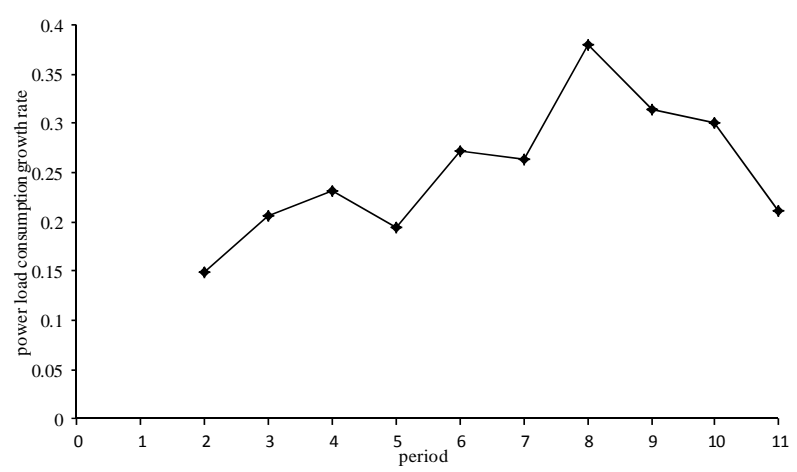

Figure 3. Power load consumption growth rate.

and high level, respectively, and the growth rate transmits in these 3 intervals. The power load of period 11 (2008 2010) is [689.719,809.903] $\times 10^{8} \mathrm{KWh}$, and the growth rate is $21.07 \%$, under the low level.

\section{Results Analysis}

In this study, the power load and the probabilities of power load in different growth states are forecasted during the three periods. Tables 1-3 present the forecasting result in different satisfaction degree (larger than 0.3, 0.6 and 0.9 ). The result of the forecasting in the three periods is in tree-like structure. There are 3 scenarios in period 1 , 9 scenarios in period 2 , and 27 scenarios in period 3 . Tables 1-3 show the probability of all the scenarios, the interval value of probability becomes narrow with the increase of satisfaction degree. Higher satisfaction degree denotes that the real transition probability close to the probability generated from the statistic data; and the lower satisfaction degree indicates that the real transition probability distributes is in a wide range around the probability gotten from statistic data. From that point, the lower satisfaction degree would lead to higher uncertainties. The satisfaction degree depends on the history statistic. If the history statistic is sufficient and the transition rule of growth date is obvious, the transition probability 
Table 1. The forecasting results of power load in period 1.

\begin{tabular}{ccccc}
\hline \multirow{2}{*}{ Growth state } & Power load $\left(10^{8} \mathrm{KWh}\right)$ & \multicolumn{3}{c}{ Probability in different satisfaction degree } \\
\cline { 3 - 4 } & & 0.3 & 0.6 & 0.9 \\
\hline $\mathrm{L}$ & {$[786.280,998.082]$} & {$[0.217,0.450]$} & {$[0.266,0.40]$} & {$[0.317,0.350]$} \\
$\mathrm{M}$ & {$[841.457,1052.874]$} & {$[0.433,0.900]$} & {$[0.33,0.800]$} & {$[0.633,0.700]$} \\
$\mathrm{H}$ & {$[896.635,1117.666]$} & 0 & 0 & 0 \\
\hline
\end{tabular}

Table 2. The forecasting results of power load in period 2.

\begin{tabular}{|c|c|c|c|c|}
\hline \multirow{2}{*}{ Growth state } & \multirow{2}{*}{ Power load (10 $\left.10^{8} \mathrm{KWh}\right)$} & \multicolumn{3}{|c|}{ Probability in different satisfaction degree } \\
\hline & & 0.3 & 0.6 & 0.9 \\
\hline $\mathrm{L}-\mathrm{L}$ & {$[896.359,1205.459]$} & {$[0.0469,0.2024]$} & {$[0.0711,0.1600]$} & {$[0.1003,0.1225]$} \\
\hline L-M & {$[959.261,1284.506]$} & {$[0.0939,0.4050]$} & {$[0.1422,0.3200]$} & {$[0.2005,0.2450]$} \\
\hline $\mathrm{L}-\mathrm{H}$ & {$[1022.163,1363.553]$} & 0 & 0 & 0 \\
\hline M-L & {$[959.261,1284.506]$} & {$[0.0939,0.4050]$} & {$[0.1422,0.3200]$} & {$[0.2005,0.2450]$} \\
\hline M-M & {$[1026.578,1368.736]$} & {$[0.0939,0.4050]$} & {$[0.1422,0.3200]$} & {$[0.2005,0.2450]$} \\
\hline $\mathrm{M}-\mathrm{H}$ & {$[1093.894,1452.966]$} & {$[0.0939,0.4050]$} & {$[0.1423,0.3201]$} & {$[0.2006,0.2451]$} \\
\hline H-L & {$[1022.163,1363.553]$} & 0 & 0 & 0 \\
\hline $\mathrm{H}-\mathrm{M}$ & [1093.894,1452.966] & 0 & 0 & 0 \\
\hline $\mathrm{H}-\mathrm{H}$ & [1165.625,1542.379] & 0 & 0 & 0 \\
\hline
\end{tabular}

Table 3. The forecasting results of power load in period3.

\begin{tabular}{|c|c|c|c|c|}
\hline \multirow{2}{*}{ Growth state } & \multirow{2}{*}{$\begin{array}{l}\text { Power load } \\
\left(10^{8} \mathrm{KWh}\right)\end{array}$} & \multicolumn{3}{|c|}{ Probability in different satisfaction degree } \\
\hline & & 0.3 & 0.6 & 0.9 \\
\hline L-L-L & {$[1021.849,1470.661]$} & {$[0.0102,0.0911]$} & {$[0.0189,0.0640]$} & {$[0.0318,0.0429]$} \\
\hline L-L-H & {$[1093.558,1567.097]$} & {$[0.0203,0.1822]$} & {$[0.0379,0.1280]$} & {$[0.0635,0.0857]$} \\
\hline L-L-H & {$[1165.266,1663.534]$} & 0 & 0 & 0 \\
\hline L-M-L & {$[1093.558,1567.097]$} & {$[0.0203,0.1822]$} & {$[0.0379,0.1280]$} & {$[0.0635,0.0857]$} \\
\hline L-M-M & {$[1170.298,1669.858]$} & {$[0.0203,0.1822]$} & {$[0.0379,0.1280]$} & {$[0.0635,0.0857]$} \\
\hline L-M-H & {$[1247.039,1772.618]$} & {$[0.0203,0.1822]$} & {$[0.0379,0.1280]$} & {$[0.0635,0.0857]$} \\
\hline L-H-L & {$[1165.266,1663.534]$} & 0 & 0 & 0 \\
\hline L-H-M & [1247.039,1772.618] & 0 & 0 & 0 \\
\hline L-H-H & [1328.812,1881.702] & 0 & 0 & 0 \\
\hline M-L-L & [1093.558,1567.097] & {$[0.0203,0.1822]$} & {$[0.0379,0.1280]$} & {$[0.0635,0.0857]$} \\
\hline M-L-M & [1170.298,1669.858] & {$[0.0469,0.3645]$} & {$[0.0759,0.2560]$} & {$[0.1270,0.1715]$} \\
\hline M-L-H & [1247.039,1772.618] & 0 & 0 & 0 \\
\hline M-M-L & [1770.298,1669.858] & {$[0.0203,0.1822]$} & {$[0.0379,0.1280]$} & {$[0.0635,0.0857]$} \\
\hline M-M-M & [1252.425,1779.357] & {$[0.0203,0.1822]$} & {$[0.0379,0.1280]$} & {$[0.0635,0.0857]$} \\
\hline M-M-H & [1334.551,1888.856] & [ 0.0234,0.2224] & {$[0.0379,0.1280]$} & {$[0.0635,0.0857]$} \\
\hline M-H-L & [1247.039,1772.618] & {$[0.0234,0.2224]$} & {$[0.0379,0.1280]$} & {$[0.0635,0.0857]$} \\
\hline M-H-M & [1334.551,1888.856] & 0 & 0 & 0 \\
\hline M-H-H & {$[1422.062,2005.093]$} & {$[0.0407,0.3646]$} & {$[0.0759,0.2561]$} & {$[0.1271,0.1715]$} \\
\hline H-L-L & [1165.266,1663.534] & 0 & 0 & 0 \\
\hline H-L-M & [1247.039,1772.618] & 0 & 0 & 0 \\
\hline H-L-H & [1328.812,1881.702] & 0 & 0 & 0 \\
\hline H-M-L & [1247.039,1772.618] & 0 & 0 & 0 \\
\hline H-M-M & [1328.812,1881.702] & 0 & 0 & 0 \\
\hline H-M-H & {$[1442.062,2005.093]$} & 0 & 0 & 0 \\
\hline H-H-L & [1328.812,1881.702] & 0 & 0 & 0 \\
\hline H-H-M & [1422.062,2005.093] & 0 & 0 & 0 \\
\hline $\mathrm{H}-\mathrm{H}-\mathrm{H}$ & [1515.312,2128.483] & 0 & 0 & 0 \\
\hline
\end{tabular}


gotten from statistic data can reflect the actual transition probability really, so high satisfaction degree should be selected. In contrast, if the uncertainty of transition probability is higher, low satisfaction degree should be chosen.

From the above tables, the probabilities of several results are 0 , and many forecasting power loads are with the same value. If those scenarios with the probability value of 0 are left out, the results obtained from the model could be simplified. In addition, the results with the same power load value could be integrated by accumulate the probability of the equal power load. Therefore, when the forecasting is for a very long term, the simplification would have advantage to make the results brief. However, there is also a disadvantage of the simplification. For example, the primary result is in tree structure, and convenient for scenario analysis. The results would become net structure when simplified, which is not convenient for decision making and analysis.

\section{Conclusions}

In this study, a fuzzy probability Markov chain is developed to forecast regional long term power load. The model can deal with the uncertainties in long term power load forecasting. The developed fuzzy probability Markov chain is applied to long term power load forecasting of Beijing. The forecasting result concludes all the power load scenarios. The data of the result is in tree-like structure. The disadvantage of the method is that the amount of data increases largely when deal with many periods. The forecasting result can be simplified by integrate the power loads with the same value. The simplification can deal with the disadvantage in some extent. But the simplification would make the data structure complex, which is disadvantage to analysis and decision making. In con- clusion, the fuzzy probability Markov chain model can reflect the vague and ambiguous during the process of power load forecasting through allowing uncertainties expressed as fuzzy parameters and discrete intervals. The forecasting result by the model is helpful for regional electricity power system managers in not only predicting a long term power load under uncertainty but also providing a basis for making multi-scenarios power generation/development plans.

\section{REFERENCES}

[1] Y. Cui, et al, "Application of Grey-Markov Prediction Model on Long-Term Load Forecasting," Modern Electric Power, Vol. 3, No. 28, 2011,pp. 38-47.

[2] C. C. Hsu and C. Y. Chen, "Regional Load Forecasting in Taiwan-Applications of Artificial Neural Networks,” Energy Conversion Management, Vol. 44, No. 12, 2003, pp. 1941-1949. doi:10.1016/S0196-8904(02)00225-X

[3] P. F. Pai and W. C. Hong, "Forecasting Regional Electricity Load Based on Recurrent Support Vector Machines with Genetic Algorithms,” Electric Power Systems Research, Vol. 74, No. 3, 2005, pp. 417-425. doi:10.1016/j.epsr.2005.01.006

[4] P. F. Pai, "Hybrid Ellipsoidal Fuzzy System in Forecasting Regional Electricity Loads,” Energy Conversion Management, 2006, Vol. 47, No. 15-16, pp. 2283-2289. doi:10.1016/j.enconman.2005.11.017

[5] L.-C. Ying and M.-C. Pan, "Using Adaptive Network Based Fuzzy Inference System to Forecast Regional Electric Loads," Energy Conversion and Management, Vol. 49, 2008, pp. 205-211.

[6] N. Ye, "A Markov Chain Model of Temporal Behavior for Anomaly Detection,” Workshop on Information Assurance and Security, 2000.

[7] T. M. Mithchel, Machine Learning, Boston, Ma: McGraw- Hill, 1997. 